\title{
A catalyst-free and easy nucleophilic addition of certain isatins to sterically hindered 2,6-di-tert-butyl-4-methylenecyclohexa-2,5- dienone
}

\author{
Andrei V. Bogdanov, ${ }^{* a}$ Sergei V. Bukharov, ${ }^{\mathrm{b}}$ Yulia N. Oludina, ${ }^{\mathrm{b}}$ Lenar I. Musin, ${ }^{\mathrm{a}}$ \\ Gulnara N. Nugumanova, ${ }^{b}$ Viktor V. Syakaev, ${ }^{a}$ and Vladimir F. Mironov ${ }^{a}$ \\ ${ }^{a}$ A.E. Arbuzov Institute of Organic and Physical Chemistry, Kazan Scientific Centre of Russian \\ Academy of Sciences, Arbuzov str. 8, Kazan 420088, Russian Federation

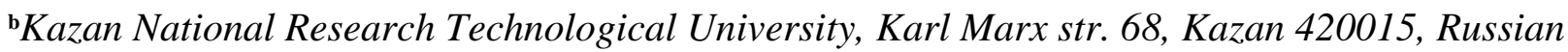 \\ Federation \\ E-mail: $\underline{\text { bogdanov@iopc.ru }}$
}

\begin{abstract}
Addition of substituted isatins to 2,6-di-tert-butyl-4-methylenecyclohexa-2,5-dienone, generated in situ from 3,5-di-tert-butyl-4-hydroxybenzyl acetate, to form 1-substituted hydroxybenzylisatins, is reported. On the basis of these isatins novel isatin-3-thiosemicarbazones as well as isoindigo derivatives bearing a 2,6-di-tert-butylphenol moiety were obtained. The structures of all novel compounds are confirmed by IR, ${ }^{1} \mathrm{H}$ NMR and ${ }^{13} \mathrm{C}$ NMR.
\end{abstract}

Keywords: Isatin, quinone methides, isoindigo, hydrazones, nucleophilic addition

\section{Introduction}

Isatin is a synthetically attractive substance due to its versatility in the chemistry of heterocycles. ${ }^{1-5}$ It is often used as a starting point in the synthesis of dyes and biologically active compounds. ${ }^{6-10}$ Isatin derivatives (Figure 1) also find applications in the field of solar energy, ${ }^{11-13}$ organic memory devices ${ }^{14}$ and organic field-effect transistors ${ }^{15,16}$

Nevertheless there are only a few works that deal with investigations of the addition reactions of isatin derivatives with multiple carbon-carbon bonds. Thus, an addition of isatin and some of its derivatives to the $\mathrm{C}=\mathrm{N}$ bond of isocyanates and $\mathrm{C}=\mathrm{C}$ bond of diphenylketene to form 1-carbamoylisatins and 1-diphenylacetylisatin respectively, have been described. ${ }^{17,18}$ The presence of an organocatalyst (triphenylphosphine (arsine), triethyl phosphite, DABCO, isocyanides) allows the addition of isatin to double carbon-carbon bonds of fumaric and acrylic esters. ${ }^{19-24}$ In all cases formation of a carbon-nitrogen bond is realized. 
<smiles>O=C1Nc2ccccc2C1=O</smiles><smiles>CCN(CC)CCN1C(=O)c2cccc(Br)c2C1=O</smiles>

$N$-Mannich bases

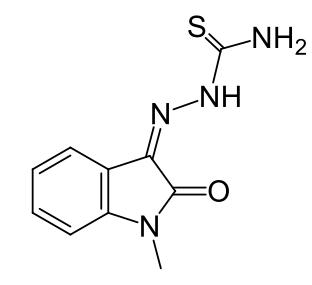

thiosemicarbazones

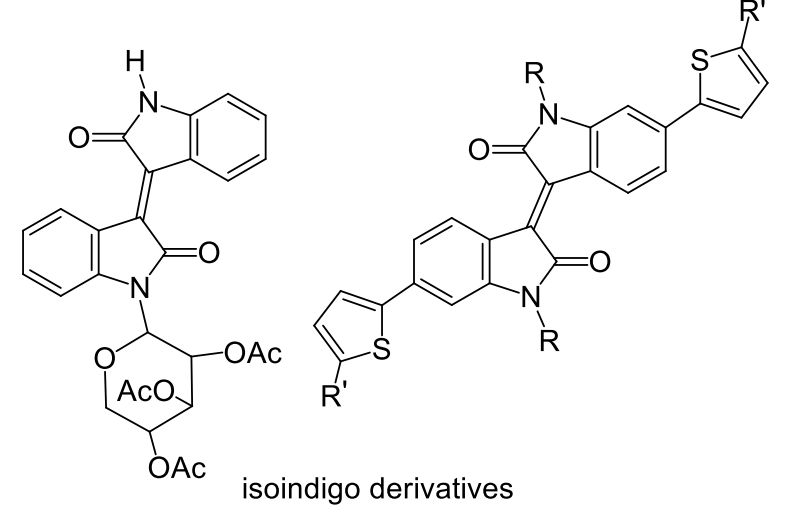

Figure 1. Isatin and its derivatives.

\section{Results and Discussion}

Herein we report the synthesis of novel isatin derivatives containing sterically hindered 2,6-ditert-butylphenol fragment. This approach is based on the condensation reaction of substituted isatins 1a-d with 3,5-di-tert-butyl-4-hydroxybenzyl acetate $\mathbf{2}$ to give corresponding benzylisatins 3a-d with high yields (Scheme 1).<smiles>[R]c1cc([R2])c2c(c1)C(=O)C(=O)N2</smiles>

1a-d<smiles>CC(=O)OCc1cc(Br)c(O)c(C(C)(C)C)c1</smiles>

2<smiles>[R10]CCN(CC)CCOC(C)=O</smiles>

3a-d

$$
\begin{aligned}
& R^{1}=B u, R^{2}=H(\mathbf{1} \mathbf{a}, \mathbf{3 a}), \\
& R^{1}=B r, R^{2}=H(\mathbf{1} \mathbf{b}, \mathbf{3 b}), \\
& R^{1}=B r, R^{2}=B r(\mathbf{1} \mathbf{c}, \mathbf{3 c}), \\
& R^{1}=H, R^{2}=\operatorname{Me}(\mathbf{1} \mathbf{d}, \mathbf{3 d})
\end{aligned}
$$

Scheme 1. Synthesis of novel sterically hindered benzylisatins 3a-d.

The reaction proceeds in dipolar aprotic solvents such as DMF or DMSO. These conditions $^{25,26}$ allows in situ generation of the highly reactive $p$-quinone methide 4 which immediately undergoes addition of corresponding isatin 1a-d with formation of a carbonnitrogen bond (Scheme 2). It should be noted here that the reaction takes place despite the hindrance due to the methyl group at the 7 position of the isatin heterocycle. 


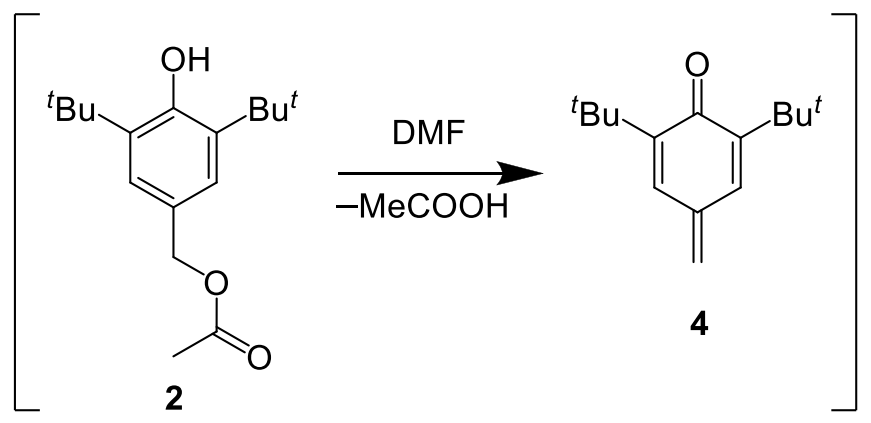

Scheme 2. In situ generation of 2,6-di-tert-butyl-4-methylenecyclohexa-2,5-dienone.

Next, on the basis of novel isatins 3a-d we succeeded in obtaining the corresponding isatin-3-thiosemicarbazides 7a-d and acylhydrazones 8a-d (Scheme 3).

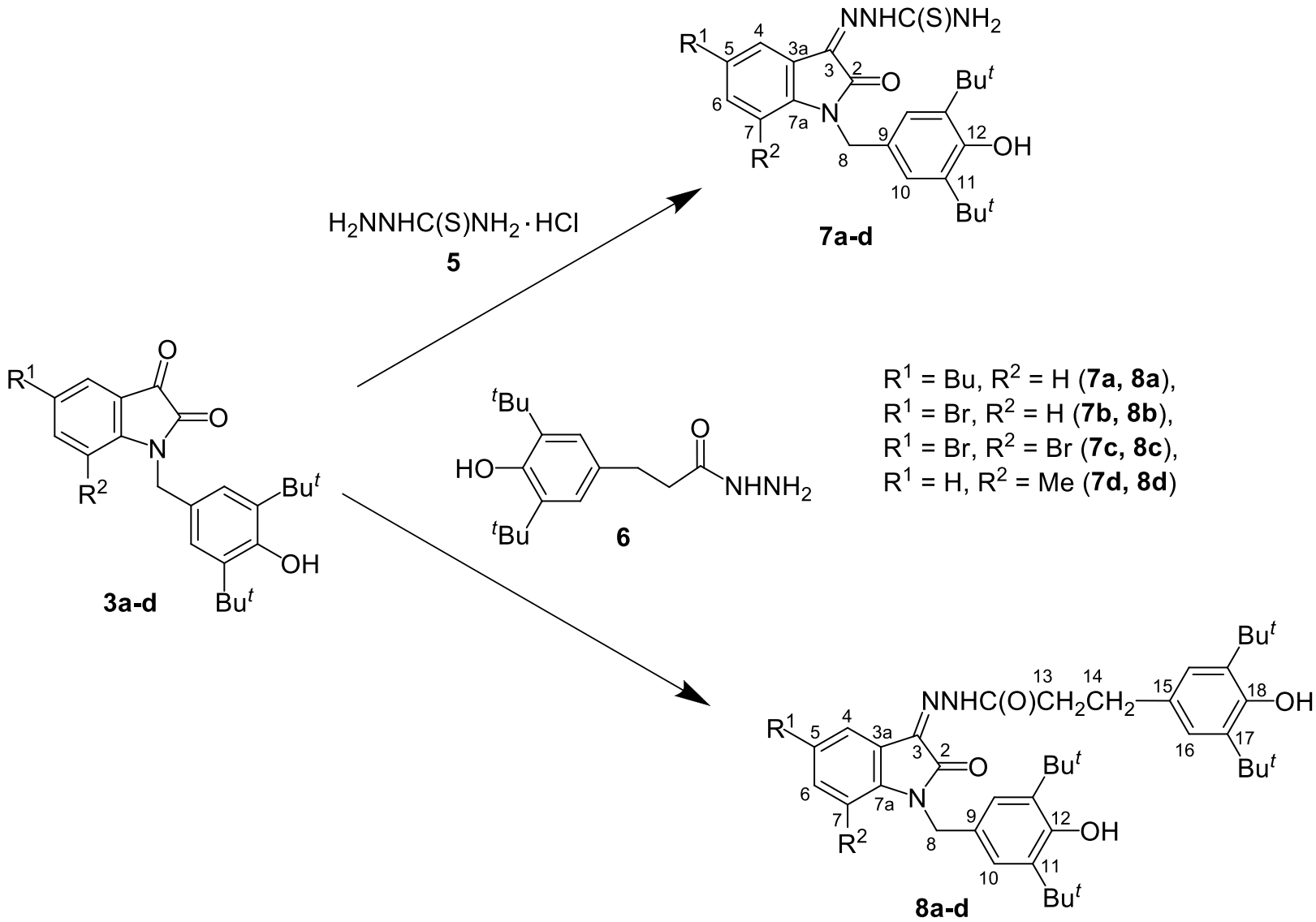

Scheme 3. Novel isatin-3-thiosemicarbazones 7a-d and acylhydrazones 8a-d.

The structures of the novel compounds 7a-d and 8a-d were determined by spectroscopic methods (IR, ${ }^{1} \mathrm{H}$ and ${ }^{13} \mathrm{C}$ NMR spectroscopy) and by elemental analyses. The presence of an NH- 
proton signal at 12-13 ppm in the ${ }^{1} \mathrm{H}$ NMR spectra of compounds $\mathbf{7}$ and $\mathbf{8}$ points to the existence of these compounds as $\mathrm{Z}_{\mathrm{C}=\mathrm{N}}$ - isomers with strong intramolecular $\mathrm{N}-\mathrm{H} \cdot \mathrm{O}$ bond. ${ }^{26}$ (Figure 2)<smiles>[X]C([R])N(C)/N=C1\C(=O)N(C[Bi])c2ccccc21</smiles>

cis, Z<smiles>[X]C([R])N(C)/N=C1\C(=O)N(C[Al])c2ccccc21</smiles>

trans, $Z$

$$
\mathrm{Ar}=4-\mathrm{HO}-3,5-\mathrm{di}^{-}{ }^{\mathrm{B}} \mathrm{BuC}_{6} \mathrm{H}_{2}
$$

Figure 2. Representation of cis, $Z$ and trans,Z-isomers of compounds 7a-d and 8a-d.

A doubling of the $\mathrm{C}(\mathrm{O}) \mathrm{CH}_{2-}, \mathrm{H}-4$ and $\mathrm{N}-\mathrm{H}$ - proton signals in ${ }^{1} \mathrm{H}$ NMR spectra of compounds 8a-d proves the presence of cis- and trans-forms regarding $\mathrm{C}(\mathrm{O})-\mathrm{N}-$ fragment $^{26}$ (Table 1). Similar doubling of NH, H-4, H-6 and $\mathrm{NH}_{2}-$ signals in ${ }^{1} \mathrm{H}$ NMR spectra also takes place for compound $\mathbf{7 c}$.

Table 1. Selected signals of cis-C(O)-N and trans-C(O)-N - forms of $\mathrm{E}_{\mathrm{C}=\mathrm{N}}-$ isomers in ${ }^{1} \mathrm{H}$ NMR spectra of compounds 8a-d

\begin{tabular}{cccccccc}
\hline \multirow{2}{*}{ Compd } & \multicolumn{2}{c}{$\mathrm{C}(\mathrm{O}) \mathrm{CH}_{2}, \delta, \mathrm{ppm}$} & \multicolumn{2}{c}{$\mathrm{H}-4, \delta, \mathrm{ppm}$} & \multicolumn{2}{c}{$\mathrm{N}-\mathrm{H}, \delta, \mathrm{ppm}$} & \multirow{2}{*}{ cis-/ trans } \\
\cline { 2 - 6 } & cis & trans & cis & trans & cis & trans & ratio \\
\hline 8a & 3.16 & 2.74 & 7.44 & 7.70 & 12.59 & 13.13 & $2.5 / 1$ \\
$\mathbf{8 b}$ & 3.12 & 2.72 & 7.69 & 7.96 & 12.51 & 13.02 & $3.8 / 1$ \\
$\mathbf{8 c}$ & 3.10 & 2.93 & 7.68 & 7.96 & 12.52 & 13.07 & $3 / 1$ \\
8d & 3.17 & 2.73 & 7.51 & 7.76 & 12.58 & 13.16 & $2.5 / 1$ \\
\hline
\end{tabular}

In a development of our investigations on the reactivity of 1,2-diketones ${ }^{27-30}$ towards trivalent phosphorus derivatives, isatins $\mathbf{3 b - d}$ were treated with tris(diethylamino)phosphine. The reaction proceeds in mild conditions and after the addition of the phosphorus reactant at $-60{ }^{\circ} \mathrm{C}$ immediately turns dark. Then on spontaneous warming to room temperature the reaction mixture become successively brown, dark-violet, dark-red and finally purple-colored, followed by precipitation of compounds $\mathbf{9 b - d}$ (Scheme 4). 

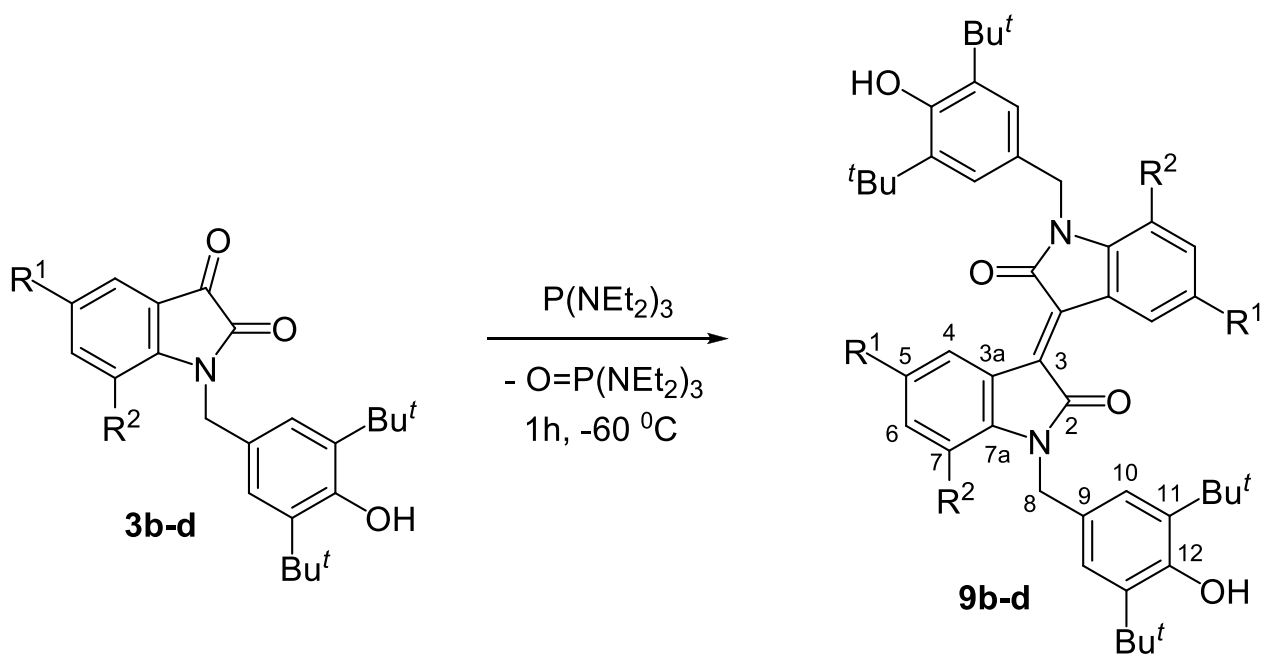

Scheme 4. Synthesis of novel sterically-hindered isoindigo derivatives $9 \mathbf{b}-\mathbf{d}$.

The structures of novel compounds 9b-d were determined using IR, ${ }^{1} \mathrm{H}$ and ${ }^{13} \mathrm{C}$ NMR spectroscopy. Thus, for example, in compound 9d the most significant observation is the downfield shift of the H-4 signal from $7.51 \mathrm{ppm}$ in the starting isatin 3d to $9.04 \mathrm{ppm}$ in the corresponding isoindigo 9d. Probably this is due to the formation of intramolecular $\mathrm{H} \cdot \mathrm{C}=\mathrm{O}$ bond. Additionally, as a result of deoxygenation and $\mathrm{C}=\mathrm{C}$ bond formation the signal of the $\mathrm{C}-3$ carbonyl carbon atom at $183.9 \mathrm{ppm}$ shifts to a signal at $133.4 \mathrm{ppm}$.

\section{Conclusions}

In summary, a synthetic method for the preparation of novel highly functionalized benzylisatins was developed. It consists in nucleophilic addition of substituted isatins to an in situ generated highly reactive $p$-quinone methide. Furthermore, this approach allows access to various isatin-3thiosemicarbazones and hydrazones as well as isoindigos which are interesting molecules for biological studies and radical-chain oxidation processes inhibitors.

\section{Experimental Section}

General. All melting points were measured with a Stuart digital SMP10 apparatus. Solvents were distilled and dried by standard literature procedures prior to use. Elemental analyses for C, $\mathrm{H}$ and $\mathrm{N}$ were performed using a CHNS-3 analyzer. IR spectra were measured with Bruker Vector-22 spectrometer as suspensions in Nujol. The ${ }^{1} \mathrm{H}$ and ${ }^{13} \mathrm{C}$ NMR spectra were recorded on a Bruker Avance-400 instrument (400 MHz for ${ }^{1} \mathrm{H}$ and $100.6 \mathrm{MHz}$ for ${ }^{13} \mathrm{C}$ ). Chemical shifts are 
given in ppm $(\delta)$ relative to residual DMSO or $\mathrm{CHCl}_{3}$ signals. Isatin derivatives 1a-d were prepared by known synthetic procedures. ${ }^{31-33}$

Preparation of $\boldsymbol{N}$-substituted 3,5-di-tert-butyl-4-hydroxybenzylindoline-2,3-diones 3a-c. A mixture of substituted isatin 1a-c $(10 \mathrm{mmol}), 3,5$-di-tert-butyl-4-hydroxybenzylacetate 2 (1.73 g, $11 \mathrm{mmol})$ and triethylamine (a few drops) in absolute DMF $(10 \mathrm{ml})$ was stirred under $70{ }^{\circ} \mathrm{C}$ for 5 $\mathrm{h}$, and cooled to r.t. Resulted solution was treated with $10 \%$ aqueous $\mathrm{NaCl}$ (200 ml). Precipitate that formed was filtered off, washed with water and air-dried.

5-Butyl-1-(3,5-di-tert-butyl-4-hydroxybenzyl)indoline-2,3-dione (3a). Dark-orange solid, yield 83\%, mp 106-107 ${ }^{\circ} \mathrm{C}$, IR: $\left(v_{\max }, \mathrm{cm}^{-1}\right): 3639(\mathrm{OH}), 1732(\mathrm{C}=\mathrm{O}), 1619,1595,1488,1436$, $1336,1285,1236,1179,1162,1124,1026,886 .{ }^{1} \mathrm{H} \mathrm{NMR}\left(\mathrm{CDCl}_{3}\right) \delta_{\mathrm{H}} 0.91\left(\mathrm{t},{ }^{3} J_{\mathrm{HH}} 8.5 \mathrm{~Hz}, 3 \mathrm{H}\right.$, $\left.\mathrm{CH}_{3}\right), 1.32\left(\mathrm{~m}, 2 \mathrm{H}, \mathrm{CH}_{2}\right), 1.40(\mathrm{~s}, 18 \mathrm{H}, t-\mathrm{Bu}), 1.55\left(\mathrm{~m}, 2 \mathrm{H}, \mathrm{CH}_{2}\right), 2.56\left(\mathrm{t}, 2 \mathrm{H}, \mathrm{CH}_{2}\right), 4.78(\mathrm{~s}, 2 \mathrm{H}$, $\left.\mathrm{CH}_{2}\right), 5.21(\mathrm{~s}, 1 \mathrm{H}, \mathrm{OH}), 6.80\left(\mathrm{~d},{ }^{3} J_{\mathrm{HH}} 8.1 \mathrm{~Hz}, 1 \mathrm{H}, \mathrm{H}-7\right), 7.16(\mathrm{~s}, 2 \mathrm{H}, \mathrm{H}-10), 7.32\left(\mathrm{dd},{ }^{3} J_{\mathrm{HH}} 8.1 \mathrm{~Hz}\right.$, $\left.{ }^{4} J_{\mathrm{HH}} 1.3 \mathrm{~Hz}, 1 \mathrm{H}, \mathrm{H}-6\right), 7.42$ (br s, $\left.1 \mathrm{H}, \mathrm{H}-4\right) .{ }^{13} \mathrm{C} \mathrm{NMR}\left(\mathrm{CDCl}_{3}\right) \delta_{\mathrm{C}} 13.8,22.1,30.2,30.9,33.3$, 34.7, 44.4, 110.7, 117.8, 124.8, 125.0, 125.5, 136.5, 138.1, 138.6, 149.2, 153.6, 158.4, 183.9. Anal. Calcd for $\mathrm{C}_{27} \mathrm{H}_{35} \mathrm{NO}_{3}$ (421.26): C, 76.92; H, 8.37; N, 3.32\%, Found: C, 76.86; H, 8.27; N, $3.18 \%$.

5-Bromo-1-(3,5-di-tert-butyl-4-hydroxybenzyl)indoline-2,3-dione (3b). Orange solid, yield 92\%, mp 178-180 ${ }^{\circ} \mathrm{C}$, IR: $\left(v_{\max }, \mathrm{cm}^{-1}\right): 3639(\mathrm{OH}), 1736(\mathrm{C}=\mathrm{O}), 1604,1331,1237,1178,1159$, 1127, 1033, 837. ${ }^{1} \mathrm{H} \mathrm{NMR}\left(\mathrm{CDCl}_{3}\right) \delta_{\mathrm{H}} 1.40(\mathrm{~s}, 18 \mathrm{H}, t-\mathrm{Bu}), 4.80\left(\mathrm{~s}, 2 \mathrm{H}, \mathrm{CH}_{2}\right), 5.24(\mathrm{~s}, 1 \mathrm{H}, \mathrm{OH})$, $6.80\left(\mathrm{~d},{ }^{3} J_{\mathrm{HH}} 8.4 \mathrm{~Hz}, 1 \mathrm{H}, \mathrm{H}-7\right), 7.12$ (s, 2H, H-10), 7.63 (dd, $\left.{ }^{3} J_{\mathrm{HH}} 8.4 \mathrm{~Hz},{ }^{4} J_{\mathrm{HH}} 2.1 \mathrm{~Hz}, 1 \mathrm{H}, \mathrm{H}-6\right)$, $7.70\left(\mathrm{~d},{ }^{4} J_{\mathrm{HH}} 2.0 \mathrm{~Hz}, 1 \mathrm{H}, \mathrm{H}-4\right) .{ }^{13} \mathrm{C} \mathrm{NMR}\left(\mathrm{CDCl}_{3}\right) \delta_{\mathrm{C}} 30.2,34.3,44.5,112.6,116.5,118.9,124.7$, 124.8, 128.1, 136.7, 140.3, 149.8, 153.8, 157.5, 182.4. Anal. Calcd for $\mathrm{C}_{23} \mathrm{H}_{26} \mathrm{BrNO}_{3}(443.11)$ : C, 62.17; H, 5.90; N, 3.15\%, Found: C, 61.96; H, 5.77; N, 3.08\%.

5,7-Dibromo-1-(3,5-di-tert-butyl-4-hydroxybenzyl)indoline-2,3-dione $\quad \mathbf{3 c})$ Bright-orange solid, yield 88\%, mp 155-157 ${ }^{\circ} \mathrm{C}$, IR: $\left(v_{\max }, \mathrm{cm}^{-1}\right): 3619(\mathrm{OH}), 1740(\mathrm{C}=\mathrm{O}), 1598,1406,1360$, 1336, 1310, 1275, 1223, 1144, 885. ${ }^{1} \mathrm{H}$ NMR $\left(\mathrm{CDCl}_{3}\right) \delta_{\mathrm{H}} 1.40(\mathrm{~s}, 18 \mathrm{H}, t$-Bu), $5.20(\mathrm{~s}, 1 \mathrm{H}, \mathrm{OH})$, $5.32\left(\mathrm{~s}, 2 \mathrm{H}, \mathrm{CH}_{2}\right), 7.21$ (s, 2H, H-10), $7.68\left(\mathrm{dd},{ }^{4} J_{\mathrm{HH}} 2.0 \mathrm{~Hz}, 1 \mathrm{H}, \mathrm{H}-6\right), 7.85\left(\mathrm{~d},{ }^{4} J_{\mathrm{HH}} 2.0 \mathrm{~Hz}, 1 \mathrm{H}\right.$, $\mathrm{H}-4) .{ }^{13} \mathrm{C} \mathrm{NMR}\left(\mathrm{CDCl}_{3}\right) \delta_{\mathrm{C}} 30.2,34.3,44.5,112.6,116.5,118.9,124.7,124.8,128.1,136.7$, 140.3, 149.8, 153.8, 157.5, 182.8. Anal. Calcd for $\mathrm{C}_{23} \mathrm{H}_{25} \mathrm{Br}_{2} \mathrm{NO}_{3}$ (521.02): C, 52.79; H, 4.82; N, $2.68 \%$, Found: C, 52.66; H, 4.70; N, 2.51\%.

7-Methyl-1-(3,5-di-tert-butyl-4-hydroxybenzyl)indoline-2,3-dione (3d). A solution of 7methylisatin 1d (909 mg, $6 \mathrm{mmol})$ and 3,5-di-tert-butyl-4-hydroxybenzylacetate 2 (1.58 g, 6 $\mathrm{mmol})$ in DMF $(50 \mathrm{ml})$ was stirred under $70{ }^{\circ} \mathrm{C}$ for $5 \mathrm{~h}$ and additionally for 5 days at r.t. Then resulted solution was treated with $10 \%$ aqueous $\mathrm{NaCl}(200 \mathrm{ml})$ followed by extraction with ether $(100 \mathrm{ml})$. Combined organic extracts was rotary evaporated to form $1.54 \mathrm{~g}$ (72\%) of compound 3d as orange solid, mp 165-168 ${ }^{\circ} \mathrm{C}$, IR: $\left(v_{\max }, \mathrm{cm}^{-1}\right): 3643(\mathrm{OH}), 1742(\mathrm{C}=\mathrm{O}), 1725(\mathrm{C}=\mathrm{O}), 1602$, $1437,1365,1345,1248,1212,1171,1155,1051,769 .{ }^{1} \mathrm{H}$ NMR $\left(\mathrm{CDCl}_{3}\right) \delta_{\mathrm{H}} 1.37(\mathrm{~s}, 18 \mathrm{H}, t$-Bu), $2.36\left(\mathrm{~s}, 3 \mathrm{H}, \mathrm{CH}_{3}\right), 5.08\left(\mathrm{~s}, 2 \mathrm{H}, \mathrm{CH}_{2}\right), 5.15(\mathrm{~s}, 1 \mathrm{H}, \mathrm{OH}), 7.00(\mathrm{~m}, 1 \mathrm{H}, \mathrm{H}-5), 7.01$ (s, 2H, H-10), $7.28\left(\mathrm{~d},{ }^{3} J_{\mathrm{HH}} 7.7 \mathrm{~Hz}, 1 \mathrm{H}, \mathrm{H}-6\right), 7.51\left(\mathrm{~d},{ }^{3} J_{\mathrm{HH}} 7.4 \mathrm{~Hz}, 1 \mathrm{H}, \mathrm{H}-4\right) .{ }^{13} \mathrm{C} \mathrm{NMR}\left(\mathrm{CDCl}_{3}\right) \delta_{\mathrm{C}} 18.8,30.2$, 
34.3, 45.5, 118.9, 122.2, 122.7, 123.5, 123.9, 126.7, 136.5, 142.4, 149.1, 153.2, 159.8, 183.9. Anal. Calcd for $\mathrm{C}_{24} \mathrm{H}_{29} \mathrm{NO}_{3}$ (379.21): C, 75.96; H, 7.70; N, 3.69\%, Found: C, 75.83; H, 7.58; N, $3.58 \%$.

General procedure for the synthesis of substituted 1-(3,5-di-tert-butyl-4-hydroxybenzyl)indoline-2,3-dione 3-thiosemicarbazones 7a-d. A mixture of substituted isatin 3a-d (10 mmol), thiosemicarbazide hydrochloride $5(75 \mathrm{mg}, 12 \mathrm{mmol})$ and triethylamine $(0.05 \mathrm{ml}, 0.4 \mathrm{mmol})$ in ethanol $(10 \mathrm{ml})$ was stirred at $80{ }^{\circ} \mathrm{C}$ for $6 \mathrm{~h}$, then cooled to r.t. The precipitate was collected by filtration, washed with ethanol $(25 \mathrm{ml})$ and air-dried to give 7.

5-Butyl-1-(3,5-di-tert-butyl-4-hydroxybenzyl)indoline-2,3-dione 3-thiosemicarbazone (7a). Yellow solid, yield 52\%, mp $208^{\circ} \mathrm{C}$, IR: $\left(v_{\max }, \mathrm{cm}^{-1}\right): 3577(\mathrm{OH}), 3413\left(\mathrm{NH}_{2}\right), 3245\left(\mathrm{NH}_{2}\right), 3157$ $(\mathrm{NH}), 1685(\mathrm{C}=\mathrm{O}), 1612(\mathrm{C}=\mathrm{N}), 1446,1310,1240,1191,1140,1127,1027,986,850 .{ }^{1} \mathrm{H}$ NMR $\left(\mathrm{CDCl}_{3}\right) \delta_{\mathrm{H}} 0.92\left(\mathrm{t},{ }^{3} J_{\mathrm{HH}} 7.4 \mathrm{~Hz}, 3 \mathrm{H}, \mathrm{CH}_{3}\right), 1.31-1.37\left(\mathrm{~m}, 2 \mathrm{H}, \mathrm{CH}_{2}\right), 1.40(\mathrm{~s}, 18 \mathrm{H}, t-\mathrm{Bu}), 1.55-$ $1.61\left(\mathrm{~m}, 2 \mathrm{H}, \mathrm{CH}_{2}\right), 2.59$ (br t, $\left.2 \mathrm{H}, \mathrm{CH}_{2}\right), 4.79$ (s, 2H, $\mathrm{CH}_{2}$ ), 5.20 (br s, $\left.1 \mathrm{H}, \mathrm{OH}\right), 6.55$ (br s, 1H, $\mathrm{NH}_{2}$ ), 6.82 (d, 1H, $\left.{ }^{3} \mathrm{~J}_{\mathrm{HH}} 8.1 \mathrm{~Hz}, \mathrm{H}-7\right), 7.14-7.15$ (m, 2H, H-10, 1H, H-6), 7.39 (br s, 1H, H-4), 7.54 (br s, $\left.1 \mathrm{H}, \mathrm{NH}_{2}\right), 12.93(\mathrm{~s}, 1 \mathrm{H}, \mathrm{NH} \cdot \mathrm{O}) .{ }^{13} \mathrm{C} \mathrm{NMR}\left(\mathrm{CDCl}_{3}\right) \delta_{\mathrm{C}} 13.9,22.2,30.2,33.7,34.3$, 35.2, 43.9, 109.9, 119.4, 120.7, 124.8, 125.8, 131.5, 132.6, 136.4, 138.0, 141.6, 153.5, 161.1, 180.1. Anal. Calcd for $\mathrm{C}_{28} \mathrm{H}_{38} \mathrm{~N}_{4} \mathrm{O}_{2} \mathrm{~S}$ (494.27): C, 67.98; H, 7.74; N, 11.33\%, Found: C, 67.71; H, 7.57; N, 11.19\%.

5-Bromo-1-(3,5-di-tert-butyl-4-hydroxybenzyl)indoline-2,3-dione 3-thiosemicarbazone (7b). Yellow solid, yield 79\%, mp 248-250 ${ }^{\circ} \mathrm{C}$ (dec.), IR: $\left(v_{\max }, \mathrm{cm}^{-1}\right): 3619(\mathrm{OH}), 3412\left(\mathrm{NH}_{2}\right), 3248$ $\left(\mathrm{NH}_{2}\right), 3172(\mathrm{NH}), 1682(\mathrm{C}=\mathrm{O}), 1599(\mathrm{C}=\mathrm{N}), 1461,1354,1328,1239,1161,1145,1124,1058$, 1031, 971, 817. ${ }^{1} \mathrm{H}$ NMR (DMSO-d $) \delta_{\mathrm{H}} 1.33(\mathrm{~s}, 18 \mathrm{H}, t-\mathrm{Bu}), 4.84\left(\mathrm{~s}, 2 \mathrm{H}, \mathrm{CH}_{2}\right), 6.93(\mathrm{~s}, 1 \mathrm{H}$, $\mathrm{OH}), 7.12$ (s, 2H, H-10), 7.14 (d, $\left.{ }^{3} J_{\mathrm{HH}} 8.3 \mathrm{~Hz} 1 \mathrm{H}, \mathrm{H}-7\right), 7.57$ (dd, ${ }^{3} J_{\mathrm{HH}} 8.4 \mathrm{~Hz},{ }^{4} J_{\mathrm{HH}} 2.0 \mathrm{~Hz}, 1 \mathrm{H}$, $\mathrm{H}-6), 7.95\left(\mathrm{~d},{ }^{4} J_{\mathrm{HH}} 2.0 \mathrm{~Hz} 1 \mathrm{H}, \mathrm{H}-4\right), 8.87\left(\mathrm{~s}, 1 \mathrm{H}, \mathrm{NH}_{2}\right), 9.15\left(\mathrm{~s}, 1 \mathrm{H}, \mathrm{NH}_{2}\right), 12.23(\mathrm{~s}, 1 \mathrm{H}$, $\mathrm{NH} \cdots \mathrm{O}) .{ }^{13} \mathrm{C}$ NMR (DMSO-d 6 ) $\delta_{\mathrm{C}} 30.2,34.4,43.0,112.3,114.8,121.6,123.3,124.1,126.4$, 129.5, 133.0, 139.4, 141.8, 153.3, 160.3, 178.8. Anal. Calcd for $\mathrm{C}_{24} \mathrm{H}_{29} \mathrm{BrN}_{4} \mathrm{O}_{2} \mathrm{~S}$ (516.12): C, 55.70; H, 5.65; N, 10.83\%, Found: C, 55.53; H, 5.42; N, 10.68\%.

5,7-Dibromo-1-(3,5-di-tert-butyl-4-hydroxybenzyl)indoline-2,3-dione 3-thiosemicarbazone (7c). Isomers ratio 3:1. Yellow solid, yield 96\%, $220 \mathrm{mg}, \mathrm{mp} 240{ }^{\circ} \mathrm{C}\left(\mathrm{dec}\right.$.), IR: $\left(v_{\max }, \mathrm{cm}^{-1}\right)$ : $3623(\mathrm{OH}), 3414\left(\mathrm{NH}_{2}\right), 3248\left(\mathrm{NH}_{2}\right), 3156(\mathrm{NH}), 1694(\mathrm{C}=\mathrm{O}), 1603(\mathrm{C}=\mathrm{N}), 1554,1463,1444$, 1343, 1320, 1237, 1151, 1127, 1074, 1038, 976, 861, 786, 725. ${ }^{1} \mathrm{H}$ NMR (DMSO-d 6 ) $\delta_{\mathrm{H}}$ (major isomer) $1.31(\mathrm{~s}, 18 \mathrm{H}, t-\mathrm{Bu}), 5.21\left(\mathrm{~s}, 2 \mathrm{H}, \mathrm{CH}_{2}\right), 6.90(\mathrm{~s}, 1 \mathrm{H}, \mathrm{OH}), 7.02(\mathrm{~s}, 2 \mathrm{H}, \mathrm{H}-10), 7.81\left(\mathrm{~d},{ }^{4} J_{\mathrm{HH}}\right.$ $1.8 \mathrm{~Hz}, 1 \mathrm{H}, \mathrm{H}-4), 8.09$ (d, $\left.{ }^{4} J_{\mathrm{HH}} 2.0 \mathrm{~Hz}, 1 \mathrm{H}, \mathrm{H}-6\right), 8.99$ (s, 1H, NH $\mathrm{NH}_{2}, 9.24$ (s, 1H, NH$)_{2}, 12.13$ (s, $1 \mathrm{H}, \mathrm{NH} \cdot \mathrm{O})$; (minor isomer) $1.31(\mathrm{~s}, 18 \mathrm{H}, t-\mathrm{Bu}), 5.21\left(\mathrm{~s}, 2 \mathrm{H}, \mathrm{CH}_{2}\right), 6.90(\mathrm{~s}, 1 \mathrm{H}, \mathrm{OH}), 7.02(\mathrm{~s}, 2 \mathrm{H}$, $\mathrm{H}-10), 7.77\left(\mathrm{~d},{ }^{4} J_{\mathrm{HH}} 1.8 \mathrm{~Hz}, 1 \mathrm{H}, \mathrm{H}-4\right), 7.91$ (d, $\left.{ }^{4} J_{\mathrm{HH}} 1.6 \mathrm{~Hz}, 1 \mathrm{H}, \mathrm{H}-6\right), 8.87$ (s, 1H, NH ), 9.16 (s, $1 \mathrm{H}, \mathrm{NH}_{2}$ ), 12.23 (s, 1H, NH·O). ${ }^{13} \mathrm{C}$ NMR (DMSO-d 6 ) $\delta_{\mathrm{C}} 30.2,34.5,43.8,103.6,115.3,122.7$, 123.1, 124.7, 127.5, 128.1, 136.9, 139.0, 139.2, 153.0, 161.1, 178.8. Anal. Calcd for $\mathrm{C}_{24} \mathrm{H}_{28} \mathrm{Br}_{2} \mathrm{~N}_{4} \mathrm{O}_{2} \mathrm{~S}$ (594.03): C, 48.33; H, 4.73; N, 9.39\%, Found: C, 48.15; H, 4.49; N, 9.18\%. 
7-Methyl-1-(3,5-di-tert-butyl-4-hydroxybenzyl)indoline-2,3-dione 3-thiosemicarbazone (7d). Yellow solid, yield $71 \%$, mp $236{ }^{\circ} \mathrm{C}$ (dec.), IR: $\left(v_{\max }, \mathrm{cm}^{-1}\right): 3631(\mathrm{OH}), 3396\left(\mathrm{NH}_{2}\right), 3291-3256$ $\left(\mathrm{NH}_{2}\right), 3155(\mathrm{NH}), 1675(\mathrm{C}=\mathrm{O}), 1604(\mathrm{C}=\mathrm{N}), 1463,1439,1357,1337,1240,1139,1106,1075$, 1004, 869, 824, 799, 743, 704, 622. ${ }^{1} \mathrm{H}$ NMR (DMSO-d $) \delta_{\mathrm{H}} 1.29$ (s, 18H, $t$-Bu), 2.29 (s, 3H, $\left.\mathrm{CH}_{3}\right), 5.08$ (s, 2H, CH ), $6.92(\mathrm{~s}, 1 \mathrm{H}, \mathrm{OH}), 6.93$ (s, 2H, H-10), 7.05 (t, $\left.{ }^{3} J_{\mathrm{HH}} 7.6 \mathrm{~Hz}, 1 \mathrm{H}, \mathrm{H}-5\right)$, 7.14 (d, $\left.{ }^{3} J_{\mathrm{HH}} 7.6 \mathrm{~Hz}, 1 \mathrm{H}, \mathrm{H}-6\right), 7.66$ (d, $\left.{ }^{3} J_{\mathrm{HH}} 7.6 \mathrm{~Hz}, 1 \mathrm{H}, \mathrm{H}-4\right), 8.77$ (s, 1H, NH 2$), 9.09$ (s, 1H, $\mathrm{NH}_{2}$ ), 12.42 (s, 1H, NH·O). ${ }^{13} \mathrm{C}$ NMR (DMSO-d 6 ) $\delta_{\mathrm{C}} 17.8,30.2,34.4,44.1,118.8,120.1,121.0$, $122.0,123.1,127.9,130.6,134.8,139.6,140.8,152.9,161.6,178.7$. Anal. Calcd for $\mathrm{C}_{25} \mathrm{H}_{32} \mathrm{~N}_{4} \mathrm{O}_{2} \mathrm{~S}$ (452.22): C, 66.34; H, 7.13; N, 12.38\%, Found: C, 66.15; H, 6.98; N, $12.05 \%$.

General procedure for the synthesis of substituted 3-(3,5-Di-tert-butyl-4-hydroxyphenyl)propionic acid [1-(3,5-di-tert-butyl-4-hydroxybenzyl)-2-oxo-1,2-dihydroindol-3-ylidene]hydrazides 8a-d. A mixture of substituted isatin 3a-d (10 mmol), 3-(3,5-di-tert-butyl-4hydroxyphenyl)propionic acid hydrazide $6(140 \mathrm{mg}, 10 \mathrm{mmol})$ and trifluoroacetic acid $(0.5 \mathrm{ml})$ in ethanol $(10 \mathrm{ml})$ was stirred at $70{ }^{\circ} \mathrm{C}$ for $5 \mathrm{~h}$, and cooled to r.t. The precipitate was filtered off, washed with ethanol $(25 \mathrm{ml})$ and air-dried to give 8a-d.

3-(3,5-Di-tert-butyl-4-hydroxyphenyl)propionic acid [5-butyl-1-(3,5-di-tert-butyl-4-hydroxybenzyl)-2-oxo-1,2-dihydroindol-3-ylidene]hydrazide (8a). Isomers ratio 2.5:1. Yellow solid, yield 45\%, mp 156-158 ${ }^{\circ} \mathrm{C}$, IR: $\left(v_{\max }, \mathrm{cm}^{-1}\right)$ : $3643(\mathrm{OH}), 3210(\mathrm{NH}), 1692(\mathrm{C}=\mathrm{O}), 1625(\mathrm{C}=\mathrm{N})$, $1611,1466,1435,1349,1319,1248,1212,1172,1154,1133,1044,1021,989,865,818,802$, 785, 739, 545. ${ }^{1} \mathrm{H} \mathrm{NMR}\left(\mathrm{CDCl}_{3}\right) \delta_{\mathrm{H}}$ (minor isomer) $0.94(\mathrm{t}, 3 \mathrm{H}, \mathrm{Me}), 1.30-1.40\left(\mathrm{~m}, 2 \mathrm{H}, \mathrm{CH}_{2}\right)$, $1.42(\mathrm{~s}, 18 \mathrm{H}, t-\mathrm{Bu}), 1.47(\mathrm{~s}, 18 \mathrm{H}, t-\mathrm{Bu}), 1.55-1.63\left(\mathrm{~m}, 2 \mathrm{H}, \mathrm{CH}_{2}\right), 2.60\left(\mathrm{t}, 2 \mathrm{H}, \mathrm{CH}_{2} \mathrm{Ar}\right), 2.74$ (br t, $2 \mathrm{H}, \mathrm{CH}_{2} \mathrm{C}(\mathrm{O})$ ), 3.00 (br t, $2 \mathrm{H}, \mathrm{ArCH}_{2} \mathrm{CH}_{2} \mathrm{C}(\mathrm{O})$ ), 4.81 (s, 2H, $\mathrm{NCH}_{2} \mathrm{Ar}$ ), 5.08 (s, 1H, OH), 5.20 (s, 1H, OH), 6.82 (d, 1H, $\left.{ }^{3} J_{\mathrm{HH}} 7.9 \mathrm{~Hz}, \mathrm{H}-7\right), 7.05-7.19$ (m, 5H, H-6, H-10, H-16), 7.70 (s, 1H, $\mathrm{H} 4), 13.13(\mathrm{~s}, 1 \mathrm{H}, \mathrm{NH} \cdot \mathrm{O}), \delta_{\mathrm{H}}$ ( major isomer ) $0.94(\mathrm{t}, 3 \mathrm{H}, \mathrm{Me}), 1.30-1.40\left(\mathrm{~m}, 2 \mathrm{H}, \mathrm{CH}_{2}\right), 1.42(\mathrm{~s}$, $18 \mathrm{H}, t$-Bu), $1.45(\mathrm{~s}, 18 \mathrm{H}, t-\mathrm{Bu}), 1.55-1.63\left(\mathrm{~m}, 2 \mathrm{H}, \mathrm{CH}_{2}\right), 2.60\left(\mathrm{t}, 2 \mathrm{H}, \mathrm{CH}_{2} \mathrm{Ar}\right), 3.00$ (br t ,2H, $\mathrm{ArCH}_{2} \mathrm{CH}_{2} \mathrm{C}(\mathrm{O})$ ), 3.16 (br t, $2 \mathrm{H}, \mathrm{CH}_{2} \mathrm{C}(\mathrm{O})$ ), 4.81 (s, 2H, NCH $\mathrm{Nr}_{2}$ ), 5.10 (s, 1H, OH), 5.20 (s, $1 \mathrm{H}, \mathrm{OH}), 6.82$ (d, 1H, $\left.{ }^{3} J_{\mathrm{HH}} 7.9 \mathrm{~Hz}, \mathrm{H}-7\right), 7.05-7.19$ (m, 5H, H-6, H-10, H-16), 7.44 (s, 1H, H-4), $12.59(\mathrm{~s}, 1 \mathrm{H}, \mathrm{NH} \cdot \mathrm{O}),{ }^{13} \mathrm{C} \mathrm{NMR}\left(\mathrm{CDCl}_{3}\right) \delta_{\mathrm{C}} 13.4,21.8,29.7,29.9,30.3,33.4,33.8,33.9,34.1$, $34.8,43.3,109.1,119.6,120.0,124.3,124.6,125.6,130.3,131.3,132.7,135.5,135.8,137.5$, 140.6, 151.7, 153.0, 160.5, 175.2. Anal. Calcd for $\mathrm{C}_{44} \mathrm{H}_{61} \mathrm{~N}_{3} \mathrm{O}_{4}(695.47): \mathrm{C}, 75.93 ; \mathrm{H}, 8.83$; N, $6.04 \%$, Found: C, 75.75; H, 8.80; N, 5.85\%.

3-(3,5-Di-tert-butyl-4-hydroxyphenyl)propionic acid [5-bromo-1-(3,5-di-tert-butyl-4hydroxybenzyl)-2-oxo-1,2-dihydroindol-3-ylidene]hydrazide (8b). Isomers ratio 3.8:1. Yellow solid, yield 85\%, mp $170{ }^{\circ} \mathrm{C}$, IR: $\left(v_{\max }, \mathrm{cm}^{-1}\right): 3647(\mathrm{OH}), 3615(\mathrm{OH}), 3213(\mathrm{NH}), 1686$ $(\mathrm{C}=\mathrm{O}), 1609$ (C=N), 1589, 1463, 1436, 1376, 1352, 1318, 1235, 1161, 1121, 1043, 984, 807, 791, 725. ${ }^{1} \mathrm{H} \mathrm{NMR}\left(\mathrm{CDCl}_{3}\right) \delta_{\mathrm{H}}$ minor isomer $1.42(\mathrm{~s}, 18 \mathrm{H}, t-\mathrm{Bu}), 1.47(\mathrm{~s}, 18 \mathrm{H}, t-\mathrm{Bu}), 2.76(\mathrm{br} \mathrm{t}$, $2 \mathrm{H}, \mathrm{CH}_{2} \mathrm{C}(\mathrm{O})$ ), 3.00 (br t, $2 \mathrm{H}, \mathrm{ArCH}_{2} \mathrm{CH}_{2} \mathrm{C}(\mathrm{O})$ ), 4.82 (s, 2H, $\mathrm{NCH}_{2} \mathrm{Ar}$ ), 5.10 (s, 1H, OH), 5.23 (s, 1H, OH), $6.80\left(\mathrm{~d}, 1 \mathrm{H},{ }^{3} \mathrm{~J}_{\mathrm{HH}} 8.2 \mathrm{~Hz}, \mathrm{H}-7\right), 7.11$ (s, 2H, H-10), 7.13 (s, 2H, H-16), 7.99 (s, 1H, $\mathrm{H}-4), 13.02(\mathrm{~s}, 1 \mathrm{H}, \mathrm{NH} \cdot \mathrm{O}), \delta_{\mathrm{H}}$ major isomer $1.42(\mathrm{~s}, 18 \mathrm{H}, t-\mathrm{Bu}), 1.47(\mathrm{~s}, 18 \mathrm{H}, t-\mathrm{Bu}), 3.14(\mathrm{br} \mathrm{t}$, 
2H, $\mathrm{CH}_{2} \mathrm{C}(\mathrm{O})$ ), 3.00 (br t, $2 \mathrm{H}, \mathrm{ArCH}_{2} \mathrm{CH}_{2} \mathrm{C}(\mathrm{O})$ ), 4.82 (s, 2H, $\mathrm{NCH}_{2} \mathrm{Ar}$ ), 5.10 (s, 1H, OH), 5.23 (s, 1H, OH), $6.80\left(\mathrm{~d}, 1 \mathrm{H},{ }^{3} J_{\mathrm{HH}} 8.2 \mathrm{~Hz}, \mathrm{H}-7\right), 7.11$ (s, 2H, H-10), 7.13 (s, 2H, H-16), 7.44 (s, 1H, $\mathrm{H}-4), 12.51$ (s, 1H, NH·O), ${ }^{13} \mathrm{C}$ NMR $\left(\mathrm{CDCl}_{3}\right) \delta_{\mathrm{C}} 29.7,29.9,30.2,33.8,33.9,34.0,43.4,110.8$, 115.4, 121.4, 123.0, 124.1, 124.5, 124.9, 130.9, 131.0, 132.7, 135.5, 136.0, 141.3, 151.7, 153.2, 160.0, 175.1. Anal. Calcd for $\mathrm{C}_{40} \mathrm{H}_{52} \mathrm{BrN}_{3} \mathrm{O}_{4}$ (717.31): C, 66.84; H, 7.29; N, 5.85\%, Found: C, $66.65 ; \mathrm{H}, 7.98 ; \mathrm{N}, 5.62 \%$.

3-(3,5-Di-tert-butyl-4-hydroxyphenyl)propionic acid [5,7-dibromo-1-(3,5-di-tert-butyl-4hydroxybenzyl)-2-oxo-1,2-dihydroindol-3-ylidene]hydrazide (8c). Isomers ratio 3:1. Yellow solid, yield 83\%, mp $218{ }^{\circ} \mathrm{C}$, IR: $\left(v_{\max }, \mathrm{cm}^{-1}\right): 3631(\mathrm{OH}), 3279(\mathrm{NH}), 1731(\mathrm{C}=\mathrm{O}), 1686(\mathrm{C}=\mathrm{O})$, $1602(\mathrm{C}=\mathrm{N}), 1553,1460,1358,1329,1269,1234,1121,1081,985,873,729 .{ }^{1} \mathrm{H} \mathrm{NMR}\left(\mathrm{CDCl}_{3}\right)$ $\delta_{\mathrm{H}}$ minor isomer $1.39(\mathrm{~s}, 18 \mathrm{H}, t-\mathrm{Bu}), 1.44(\mathrm{~s}, 18 \mathrm{H}, t-\mathrm{Bu}), 2.71$ (br s, $2 \mathrm{H}, \mathrm{CH}_{2} \mathrm{C}(\mathrm{O})$ ), 3.00 (br t, $\left.2 \mathrm{H}, \mathrm{ArCH}_{2} \mathrm{CH}_{2} \mathrm{C}(\mathrm{O})\right), 5.08(\mathrm{~s}, 1 \mathrm{H}, \mathrm{OH}), 5.17$ (s, 1H, OH), 5.31 (s, 2H, NCH $\mathrm{Nr}_{2}$ ) 7.07 (s, 2H, H10), 7.18 (s, 2H, H-16), 7.64 (s, 1H, H-4); 7.68 (s, 1H, H-6), 13.00 (s, trans-Z, 1H, NH O); $\delta_{\mathrm{H}}$ major isomer $1.39(\mathrm{~s}, 18 \mathrm{H}, t-\mathrm{Bu}), 1.44\left(\mathrm{~s}, 18 \mathrm{H}, t\right.$-Bu), $3.10\left(\mathrm{br} \mathrm{s}, 2 \mathrm{H}, \mathrm{CH}_{2} \mathrm{C}(\mathrm{O})\right), 3.00$ (br t, $2 \mathrm{H}$, $\left.\mathrm{ArCH}_{2} \mathrm{CH}_{2} \mathrm{C}(\mathrm{O})\right), 5.08$ (s, 1H, OH), 5.17 (s, 1H, OH), 5.31 (s, 2H, NCH $\mathrm{N}_{2} \mathrm{Ar}$ ), 7.07 (s, 2H, H-10), 7.18 (s, 2H, H-16), 7.64 (s, 1H, H-4); 7.69 (s, 1H, H-6), 12.44 (s, cis-Z, 1H, NH O). Due to the very low solubility of this compound in a wide range of organic solvents ${ }^{13} \mathrm{C}$ NMR spectrum could not be recorded. Anal. Calcd for $\mathrm{C}_{40} \mathrm{H}_{51} \mathrm{Br}_{2} \mathrm{~N}_{3} \mathrm{O}_{4}$ (795.22): C, 60.23; H, 6.44; N, 5.27\%, Found: C, 60.07; H, 6.28; N, 5.19\%.

3-(3,5-Di-tert-butyl-4-hydroxyphenyl)propionic acid [7-methyl-1-(3,5-di-tert-butyl-4hydroxybenzyl)-2-oxo-1,2-dihydroindol-3-ylidene]hydrazide (8d). Isomers ratio 2.5:1. Yellow solid, yield 71\%, mp 218-220 ${ }^{\circ} \mathrm{C}$, IR: $\left(v_{\max }, \mathrm{cm}^{-1}\right): 3627(\mathrm{OH}), 3285(\mathrm{NH}), 1713(\mathrm{C}=\mathrm{O})$, $1679(\mathrm{C}=\mathrm{O}), 1596(\mathrm{C}=\mathrm{N}), 1456,1436,1374,1360,1325,1256,1234,1177,1146,1119,1092$, $1029,875,798,741,509 .{ }^{1} \mathrm{H}$ NMR $\left(\mathrm{CDCl}_{3}\right) \delta_{\mathrm{H}}$ minor isomer $1.38(\mathrm{~s}, 18 \mathrm{H}, t$-Bu), 1.44 (s, trans$Z, 18 \mathrm{H}, t-\mathrm{Bu}$ ), 2.38 (s, 3H, Me), 2.73 (br t, $2 \mathrm{H}, \mathrm{CH}_{2} \mathrm{C}(\mathrm{O})$ ), 3.01 (br t, $2 \mathrm{H}, \mathrm{ArCH}_{2} \mathrm{CH}_{2} \mathrm{C}(\mathrm{O})$ ), 5.08 (s, 1H, OH), 5.12 (s, 2H, NCH $2 \mathrm{Ar}), 5.16(\mathrm{~s}, 1 \mathrm{H}, \mathrm{OH}), 6.95-7.15$ (m, 6H, H-5, H-6, H-10, H-16), 7.75 (d, trans-Z, $\left.1 \mathrm{H},{ }^{3} J_{\mathrm{HH}} 7.3 \mathrm{~Hz}, \mathrm{H}-4\right), 13.16$ (s, trans-Z, $\left.1 \mathrm{H}, \mathrm{NH} \cdot \mathrm{O}\right)$ ) major isomer $1.38(\mathrm{~s}, 18 \mathrm{H}$, $t$-Bu), 1.47 (s, cis- $Z, 18 \mathrm{H}, t$-Bu), 2.40 (s, 3H, Me), 3.17 (br t, 2H, CH $\mathrm{C}_{2} \mathrm{C}(\mathrm{O})$ ), 3.01 (br t, 2H, $\left.\mathrm{ArCH}_{2} \mathrm{CH}_{2} \mathrm{C}(\mathrm{O})\right), 5.10(\mathrm{~s}, 1 \mathrm{H}, \mathrm{OH}), 5.12$ (s, 2H, NCH$\left.{ }_{2} \mathrm{Ar}\right), 5.16(\mathrm{~s}, 1 \mathrm{H}, \mathrm{OH}), 6.95-7.15(\mathrm{~m}, 6 \mathrm{H}$, H-5, H-6, H-10, H-16), 7.51 (d, cis- $\left.Z, 1 \mathrm{H},{ }^{3} J_{\mathrm{HH}} 7.3 \mathrm{~Hz}, \mathrm{H}-4\right), 12.58$ (s, cis- $\left.Z, 1 \mathrm{H}, \mathrm{NH} \cdot \mathrm{O}\right),{ }^{13} \mathrm{C}$ NMR $\left(\mathrm{CDCl}_{3}\right) \delta_{\mathrm{C}} 18.3,29.7,29.9,30.1,33.8,33.9,34.0,44.3,118.0,120.3,120.4,122.3,122.7$, $124.5,126.8,131.2,132.2,134.4,135.5,135.9,140.7,151.7,152.7,161.4,175.1$. Anal. Calcd for $\mathrm{C}_{41} \mathrm{H}_{55} \mathrm{~N}_{3} \mathrm{O}_{4}$ (653.42): C, 75.31; H, 8.48; N, 6.43\%, Found: C, 75.09; H, 8.28; N, 6.29\%.

5,5'-Dibromo-1,1'-(3,5-di-tert-butyl-4-hydroxybenzyl)-1H,1'H-[3,3']-biindolylidene-2,2'-

dione (9b). Dark-cherry solid, yield 91\%, $437 \mathrm{mg}, \mathrm{mp}>300{ }^{\circ} \mathrm{C}, \mathrm{IR}:\left(v_{\max }, \mathrm{cm}^{-1}\right): 3616(\mathrm{OH})$, $1695(\mathrm{C}=\mathrm{O}), 1605(\mathrm{C}=\mathrm{C}), 1300,1213,1157,1120,801 .{ }^{1} \mathrm{H} \mathrm{NMR}\left(\mathrm{CDCl}_{3}\right) \delta_{\mathrm{H}} 1.32(\mathrm{~s}, 18 \mathrm{H}, t$ $\mathrm{Bu}), 4.90\left(\mathrm{~s}, 2 \mathrm{H}, \mathrm{CH}_{2}\right), 6.92$ (br s, $\left.1 \mathrm{H}, \mathrm{OH}\right), 7.11$ (d, $\left.{ }^{3} \mathrm{~J}_{\mathrm{HH}} 8.6 \mathrm{~Hz}, 1 \mathrm{H}, \mathrm{H}-7\right), 7.14$ (s, 2H, H-10), $7.64\left(\mathrm{dd},{ }^{3} J_{\mathrm{HH}} 8.5 \mathrm{~Hz},{ }^{4} J_{\mathrm{HH}} 1.6 \mathrm{~Hz}, 1 \mathrm{H}, \mathrm{H}-6\right), 9.36\left(\mathrm{~d},{ }^{4} J_{\mathrm{HH}} 1.6 \mathrm{~Hz}, 1 \mathrm{H}, \mathrm{H}-4\right)$. Anal. Calcd for $\mathrm{C}_{46} \mathrm{H}_{52} \mathrm{Br}_{2} \mathrm{~N}_{2} \mathrm{O}_{4}$ (854.23): C, 64.49; H, 6.12; N, 3.27\%, Found: C, 64.28; H, 6.01; N, 3.18\%. Due 
to the very low solubility of this compound in a wide range of organic solvents ${ }^{13} \mathrm{C}$ NMR spectrum could not be recorded.

5,5',7,7'-Tetrabromo-1,1'-(3,5-di-tert-butyl-4-hydroxybenzyl)-1H,1'H-[3,3']-biindolylidene2,2'-dione (9c). Light-purple solid, yield 83\%, $215 \mathrm{mg}, \mathrm{mp}>300{ }^{\circ} \mathrm{C}$, IR: $\left(v_{\max }, \mathrm{cm}^{-1}\right): 3438$ $(\mathrm{OH}), 1700(\mathrm{C}=\mathrm{O}), 1608(\mathrm{C}=\mathrm{C}), 1550,1333,1214,1149,1109,1023 .{ }^{1} \mathrm{H}$ NMR (DMSO-d 6$) \delta_{\mathrm{H}}$ $1.40(\mathrm{~s}, 18 \mathrm{H}, t-\mathrm{Bu}), 5.15$ (br s, $1 \mathrm{H}, \mathrm{OH}), 5.41\left(\mathrm{~s}, 2 \mathrm{H}, \mathrm{CH}_{2}\right), 7.20$ (s, 2H, H-10), 7.67 (d, ${ }^{4} J_{\mathrm{HH}} 1.8$ $\mathrm{Hz}, 1 \mathrm{H}, \mathrm{H}-6), 9.37$ (d, $\left.{ }^{4} J_{\mathrm{HH}} 1.8 \mathrm{~Hz}, 1 \mathrm{H}, \mathrm{H}-4\right) .{ }^{13} \mathrm{C}$ NMR (DMSO-d 6 ) $\delta_{\mathrm{C}} 30.3,34.4,44.9,102.5$, 115.1, 124.3, 125.8, 127.2, 131.4, 133.1, 136.0, 140.5, 141.2, 153.2, 168.0. Anal. Calcd for $\mathrm{C}_{46} \mathrm{H}_{50} \mathrm{Br}_{4} \mathrm{~N}_{2} \mathrm{O}_{4}$ (1010.05): C, 54.46; H, 4.97; N, 2.76\%, Found: C, 54.28; H, 4.91; N, $2.60 \%$.

7,7'-Dimethyl-1,1'-(3,5-di-tert-butyl-4-hydroxybenzyl)-1H,1'H-[3,3']-biindolylidene-2,2' -

dione (9d). Dark-cherry crystals, yield 85\%, $478 \mathrm{mg}, \mathrm{mp} 125{ }^{\circ} \mathrm{C}$, IR: $\left(v_{\max }, \mathrm{cm}^{-1}\right): 3460(\mathrm{OH})$, $1691(\mathrm{C}=\mathrm{O}), 1600(\mathrm{C}=\mathrm{C}), 1377,1235,1209,1187,1160,1117,1023,787 .{ }^{1} \mathrm{H} \mathrm{NMR}\left(\mathrm{CDCl}_{3}\right) \delta_{\mathrm{H}}$ $1.36(\mathrm{~s}, 18 \mathrm{H}, t-\mathrm{Bu}), 2.36\left(\mathrm{~s}, 3 \mathrm{H}, \mathrm{CH}_{3}\right), 5.18\left(\mathrm{~s}, 2 \mathrm{H}, \mathrm{CH}_{2}\right), 6.90\left(\mathrm{t},{ }^{3} J_{\mathrm{HH}} 7.8 \mathrm{~Hz}, 1 \mathrm{H}, \mathrm{H}-5\right), 7.01(\mathrm{~s}$, $2 \mathrm{H}, \mathrm{H}-10), 7.04$ (d, $\left.{ }^{3} J_{\mathrm{HH}} 7.4 \mathrm{~Hz}, 1 \mathrm{H}, \mathrm{H}-6\right), 9.04\left(\mathrm{~d},{ }^{3} J_{\mathrm{HH}} 7.8 \mathrm{~Hz}, 1 \mathrm{H}, \mathrm{H}-4\right) .{ }^{13} \mathrm{C} \mathrm{NMR}\left(\mathrm{CDCl}_{3}\right) \delta_{\mathrm{C}}$ 19.2, 30.2, 34.3, 45.5, 119.1, 122.0, 122.6, 125.9, 127.3, 128.0, 133.4, 136.2, 136.6, 142.9, 152.9, 169.1. Anal. Calcd for $\mathrm{C}_{48} \mathrm{H}_{58} \mathrm{~N}_{2} \mathrm{O}_{4}$ (726.44): C, 79.30; H, 8.04; N, 3.85\%, Found: C, 79.18; H, $7.95 ; \mathrm{N}, 3.68 \%$.

\section{Acknowledgements}

We would like to express our gratitude to Prof. Alexander R. Burilov for the discussion and valuable recommendations.

\section{References}

1. Sumpter, W. C. Chem. Rev. 1944, 34, 393

http://dx.doi.org/10.1021/cr60109a003

2. Popp, F. D. Adv. Heterocycl. Chem. 1975, 18, 1 http://dx.doi.org/10.1016/S0065-2725(08)60127-0

3. da Silva, J. F. M.; Garden, S. J.; Pinto, A. C. J. Braz. Chem. Soc. 2001, 12, 273. http://dx.doi.org/10.1590/S0103-50532001000300002

4. Lashgari, N.; Ziarani,Gh. M. Arkivoc 2012, (i), 277.

5. Singh, G. S.; Desta, Z. Y. Chem. Rev. 2012, 112, 6104. http://dx.doi.org/10.1021/cr300135y PMid:22950860

6. Karapetyan, G.; Chakrabarty, K.; Hein, M.; Langer P. Chem. Med. Chem. 2011, 6, 25. http://dx.doi.org/10.1002/cmdc.201000374 PMid:21108279 
7. Raja Solomon, V.; Hu, C.; Lee, H. Bioorg. Med. Chem. 2009, 17, 7585. http://dx.doi.org/10.1016/j.bmc.2009.08.068 PMid:19804979

8. Wee, X. K.; Yang, T.; Go, M. L. Chem. Med. Chem. 2012, 7, 777. http://dx.doi.org/10.1002/cmdc.201200018 PMid:22416043

9. Sassatelli, M.; Bouchikhi, F.; Aboab, B.; Anizon, F.; Fabbro, D.; Prudhomme, M.; Moreau, P. Anticancer Drugs 2007, 18, 1069. http://dx.doi.org/10.1097/CAD.0b013e328182d281 PMid:17704657

10. Romagnoli, R.; Baraldi, P. G.; Cruz-Lopez, O.; Preti, D.; Bermejo, J.; Estevez, F. Chem. Med. Chem. 2009, 4, 1668.

http://dx.doi.org/10.1002/cmdc.200900245

PMid:19670209

11. Walker, B.; Kim, Ch,; Nguyen, Th.-Q. Chem. Mater. 2011, 23, 470. http://dx.doi.org/10.1021/cm102189g

12. Mishra, A.; Bauerle, P. Angew. Chem. Int. Ed. 2012, 51, 2020. http://dx.doi.org/10.1002/anie.201102326 PMid:22344682

13. Zhang, G.; Fu, Y.; Xie, Zh.; Zhang, Q. Macromolecules 2011, 44, 1414. http://dx.doi.org/10.1021/ma102357b

14. Xu, X.; Li, L.; Liu, B.; Zou,Y. Appl. Phys. Lett. 2011, 98, 063303. http://dx.doi.org/10.1063/1.3554756

15. Lei, T.; Cao, Y.; Fan, Y.; Liu, Ch.-J.; Yuan, S.-Ch.; Pei, J. J. Am. Chem. Soc. 2011, 133, 6099.

http://dx.doi.org/10.1021/ja111066r

PMid:21466199

16. Ashraf, R. Sh.; Kronemeijer, A. J.; James, D. I.; Sirringhaus, H.; McCulloch, I. Chem. Commun. 2012, 48, 3939.

http://dx.doi.org/10.1039/c2cc30169e

PMid:22422164

17. Yamagishi, M.; Yamada, Y.; Ozaki, K.; Asao, M.; Shimizu, R.; Suzuki, M.; Matsumoto, M.; Matsuoka, Y.; Matsumoto, K. J. Med. Chem. 1992, 35, 2085. http://dx.doi.org/10.1021/jm00089a021

18. Singh, G. S.; Masutlha, L. L. Proc. Natl. Acad. Sci., India, Sect. A Phys. Sci. 2012, 82, 147.

19. Baharfar, R.; Tajbakhsh, M.; Hamedaninejad, A.; Hosseini, S. J. Chin. Chem. Lett. 2008, $19,175$. http://dx.doi.org/10.1016/j.cclet.2007.12.014

20. Yavari, I.; Hossaini, Z.; Karimi, E. Monatsh. Chem. 2007, 138, 1267. http://dx.doi.org/10.1007/s00706-007-0711-5 
21. Imanzadeh, G. H.; Mollaei Tavana, M.; Zamanloo, M. R.; Mansoori, Y. Chinese J. Chem. 2009, 27, 389. http://dx.doi.org/10.1002/cjoc.200990064

22. Imanzadeh, G. H.; Soltanizadeh, Z.; Khodayari, A.; Zamanloo, M.; Mansoori, Y.; Salehzadeh, J. Chinese J. Chem. 2012, 30, 891.

http://dx.doi.org/10.1002/cjoc.201100351

23. Bayat, M.; Imanieh, H.; Hossieninejad, E. Synth. Commun. 2008, 38, 2567. http://dx.doi.org/10.1080/00397910802219213

24. Maghsoodlou, M. T.; Heydari, R.; Habibi-Khorassani, S. M.; Hazeri, N.; Lashkari, M.; Rostamizadeh, M.; Sajadikhah, S. S. Synth. Commun. 2011, 41, 569. http://dx.doi.org/10.1080/00397911003629432

25. Nugumanova, G. N.; Bukharov, S.V.; Tagasheva, R. G.; Kurapova, M. V.; Mukmeneva, N. A.; Gurevich, P. A.; Burilov, A. R. Russ. J. Org. Chem. 2007, 43, 1797. http://dx.doi.org/10.1134/S1070428007120093

26. Nugumanova, G. N.; Tagasheva, R. G.; Bukharov, S.V.; Krivolapov, D. B.; Litvinov, I. A.; Syakaev, V. V.; Mukmeneva, N. A.; Burilov, A. R. Russ. Chem. Bull. 2009, 58, 1934. http://dx.doi.org/10.1007/s11172-009-0264-3

27. Bogdanov, A. V.; Mironov, V. F.; Buzykin, B. I.; Konovalov, A. I. Russ. J. Gen. Chem. 2005, 75, 825 . http://dx.doi.org/10.1007/s11176-005-0325-8

28. Bogdanov, A. V.; Mironov, V. F.; Musin, L.I.; Musin, R.Z. Synthesis 2010, 19, 3268. http://dx.doi.org/10.1055/s-0030-1258219

29. Bogdanov, A. V.; Mironov, V. F.; Musin, L.I.; Musin, R.Z.; Krivolapov, D. B.; Litvinov, I. A. Monatsh. Chem. 2011, 142, 81. http://dx.doi.org/10.1007/s00706-010-0416-Z

30. Bogdanov, A. V.; Mironov, V. F.; Musin, L.I.; Musin, R.Z.; Krivolapov, D. B.; Litvinov, I. A. Synth. Commun. 2012, 42, 2388. http://dx.doi.org/10.1080/00397911.2011.558232

31. Bolotin, B. M.; Zeryukina, L. S.; Safina, R. U.; Egorkin, V. V.; Kuliev, R. I. Chem. Het. Comp. 1981, 17, 996.

32. Vine, K. L.; Locke, J.M.; Ranson, M.; Pyne, S. G.; Bremner, J. B. Bioorg. Med. Chem. 2007, 15, 931 . http://dx.doi.org/10.1016/j.bmc.2006.10.035 PMid:17088067

33. Sandmeyer, T. Helv. Chim. Acta 1919, 2, 239. http://dx.doi.org/10.1002/hlca.19190020125 